\title{
Implications of the Visible and X-Ray Counterparts to GRB 970228
}

\author{
J. I. Katz, ${ }^{1,2}$ T. Piran, ${ }^{1}$ and R. Sari ${ }^{1}$ \\ ${ }^{1}$ The Racah Institute for Physics, The Hebrew University, Jerusalem, 91904 Israel \\ ${ }^{2}$ Department of Physics and McDonnell Center for the Space Sciences, Washington University, St. Louis, Missouri 63130
}

(Received 23 April 1997; revised manuscript received 31 December 1997)

\begin{abstract}
We propose that the visible and x-ray emission associated with the cosmic gamma-ray burst GRB 970228 but following it by hours and days was produced by a weaker continuation of the processes which produced the gamma-ray burst itself. This hypothesis predicts an instantaneous spectrum $F_{\nu} \propto \nu^{-1 / 2}$, resulting from radiative cooling of synchrotron-emitting electrons, at frequencies from the infrared to $x$ rays and higher. The limited data support this prediction. [S0031-9007(98)05397-6]
\end{abstract}

PACS numbers: $98.70 . \mathrm{Rz}$

The gamma-ray burst GRB 970228 [1,2] has been observed after a delay of 8-17 hours in $\mathrm{x}$ rays [3] and of 17 hours in visible light [4]. This marks the first detection of emission at lower frequencies following the gamma-ray observation of a gamma-ray burst (GRB) and the first detection of any visible counterpart to a GRB. We consider possible delayed visible and $\mathrm{x}$-ray emission mechanisms, and conclude that the activity by the source of the GRB continued at a much reduced intensity for at least a day. There are hints of such continued activity in other GRB, and future observations can decide if this is true of GRB in general. The observed simultaneous multiband spectrum of GRB 970228 agrees with the predictions of relativistic shock theory when the flux is integrated over a time longer than that required for a radiating electron to lose its energy.

Several mechanisms for the continuing $\mathrm{x}$-ray emission of GRB 970228 should be considered. The simplest possibility is that the relativistic particles required to explain a GRB will collide with a surrounding dilute medium. This process has been suggested [5] as the source of the gamma-ray emission itself. These models face, however, a serious problem. The observed duration of $\mathrm{x}$-ray emission [3] is roughly 1000 times the reported gamma-ray duration [1], despite a ratio of only $\sim 50$ in the observed frequency $\nu_{\text {obs }}$. Most models of this type predict a much steeper decrease in frequency as a function of time. A specific calculation [6] predicts, for example, a time scale of emission $\propto \nu_{\text {obs }}^{-5 / 12}$. One can consider, alternatively, models in which hot electrons cool, and emit lower energy photons. These models face the same problem. For example, a model [7] in which relativistic electrons radiate their energy in a constant magnetic field predicts a time scale $\propto \nu_{\mathrm{obs}}^{-1 / 2}$. Another alternative model of the x-ray emission, thermal bremsstrahlung (as in a supernova remnant), may also be excluded because the required power $\sim 10^{45} \mathrm{erg} / \mathrm{s}$ (at a cosmological redshift of a few tenths) would require an unachievable particle density $>10^{10} \mathrm{~cm}^{-3}$ even if the maximum plausible mass of $1 M_{\odot}$ is radiating.
Instead, we suggest that the observed brief intense gamma-ray emission of a GRB is only the "tip of an iceberg"; it emits gamma rays at a much lower level for time of order a day following (and perhaps preceding) the intense outburst. GRB detectors necessarily have high backgrounds because they must have very broad angular acceptance; these high backgrounds, lack of angular discrimination, and necessarily short integration times imply high thresholds for detected flux, making the continuing weak gamma-ray emission difficult to observe. The x-ray and visible radiation is then produced by the same mechanism as the gamma rays, simultaneously with their continuing emission. It is not possible to predict how rapidly this continuing emission fades, but the finiteness of the total fluence requires that, on average, it decay faster than the reciprocal of the time since the outburst. It is also possible that continuing activity produces emission that peaks in $\mathrm{x}$ rays, and does not emit gamma rays. This would not affect the predicted spectrum at $\mathrm{x}$-ray frequencies and below.

There is independent evidence for continuing gammaray activity in GRB, with durations longer than the usual values <1000 s [8]. GRB 940217 was observed [9] to emit energetic gamma rays over a duration of $\approx 5000 \mathrm{~s}$. The group of four GRB observed $[10,11]$ on 27-29 October 1996, apparently from a common source, may equally well be described as repeating bursts or as a single burst lasting two days with brief periods of high intensity amidst a much longer period of undetectably low intensity. The occasional observation of "precursors" some time before the peak emission of a GRB [12] may also indicate a longer period of weak activity.

The hypothesis that many GRB last a day or more is consistent with the demonstration [13] that the observed complex time structure of GRB on scales of seconds must be attributed to variations in the power of their energy source. In some models of the central engine [14] durations of a day are no less plausible than durations of tens of seconds.

Our suggestion that the $\mathrm{x}$-ray and visible emission of GRB 970228 was the consequence of continuing activity by the object which produced the GRB means that 
continuing gamma-ray emission may be observable in similar bursts by suitable instruments. It also leads to a specific prediction for its spectrum, which may be roughly tested with the data at hand. The instantaneous spectrum of a relativistic shock is predicted $[15,16]$ to be $F_{\nu} \propto \nu^{1 / 3}$. The spectrum integrated over the radiative decay of the electrons' energy is predicted [17] to be $F_{\nu} \propto \nu^{-1 / 2}$. Observations during the brief phases of GRB during which the Burst and Transient Source Experiment (BATSE) on the Compton Gamma Ray Observatory obtained data have shown soft gamma-ray spectra between these limits, with $F_{\nu} \propto \nu^{-1 / 2}$ found when the data are integrated over many subpulses, allowing time for shock-heated electrons to radiate their energy [17]. This prediction should be applicable to x-ray [3] and visible [4] data obtained over much longer periods of integration.

The data [3,4] from GRB 970228 are collected in Fig. 1. The $B$ and $R$ band data were obtained [4] $17 \mathrm{~h}$ after the observed GRB, and the $2-10 \mathrm{KeV} x$-ray data [3] were integrated over the period 8-17 h after the GRB. Because these data were obtained nearly simultaneously, they form a nearly instantaneous spectrum and may be directly compared. The solid line shows the predicted $F_{\nu} \propto \nu^{-1 / 2}$ slope, fitted to a weighted mean of the $B$ and $R$ data points. The $B, R$, and $\mathrm{x}$-ray data are all consistent with the predicted slope, confirming the hypothesis.

At yet lower frequencies the spectral slope is predicted to change from $F_{\nu} \propto \nu^{-1 / 2}$ to $F_{\nu} \propto \nu^{1 / 3}$, with the break occurring at the characteristic synchrotron frequency of the electrons which have undergone radiative cooling. This frequency is model dependent, but scaling from the width of the gamma-ray peak [2] suggests values $\sim 10^{11}-10^{12} \mathrm{~Hz}$ after a day.

This model predicts that if sufficiently sensitive gammaray observations are made of persistent emission from GRB it will lie along an extrapolation of the $F_{\nu} \propto \nu^{-1 / 2}$

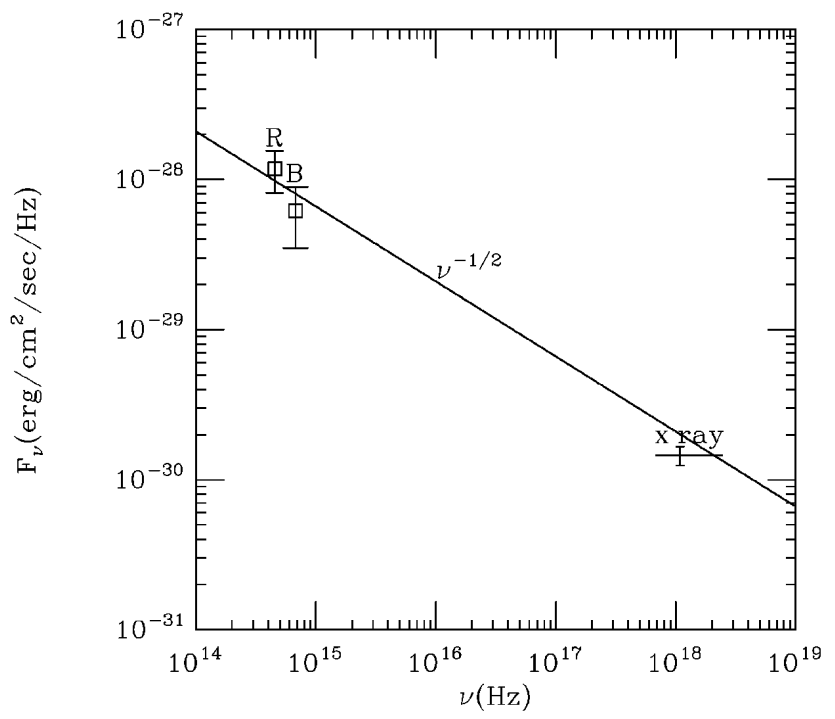

FIG. 1. Fluxes of GRB 970228 in x-ray [3], $B$ [4], and $R$ [4] bands. The straight line has the predicted $-1 / 2$ slope, normalized to the visible data. spectrum. In addition, just as the gamma-ray intensity of GRB fluctuates irregularly on all observed time scales, often with several distinct peaks, so (by analogy and by comparison to the 27-29 October 1996 bursts) should fluctuate the intensity at longer wavelengths. This may be tested by examining photon time of arrival statistics.

Following the submission of the original version of this paper, persistent emission from GRB 970508 was observed. Its visible intensity was roughly constant for nearly a day, before rising sharply to a maximum and then declining [18]. Very similar behavior was observed in $x$ rays [19]. During this first day the visible and $x$-ray fluxes fit a spectral slope $F_{\nu} \propto \nu^{-0.6}$, close to (and perhaps consistent with, allowing for the fact that data in the different bands were not obtained quite simultaneously) the predicted exponent of $-1 / 2$. The time dependence, most particularly the increase in x-ray flux, also supports our hypothesis that at least the first day of the visible and $\mathrm{x}$-ray emission was the consequence of continuing and fluctuating activity like that which produced the GRB itself, rather than an "afterglow" produced by a distinct process. The fact that several other GRB have failed to show persistent visible counterparts also suggests that this is associated with the gamma-ray emission, which is known to vary widely in temporal behavior from burst to burst, rather than with a universal and unavoidable process like collision with a surrounding medium.

We thank NASA, NSF, and the U.S.-Israel BSF for support. J. I. K. thanks Washington University for the grant of sabbatical leave and the Hebrew University for hospitality.

[1] E. Costa et al., IAU Circ., 6572 (1997).

[2] D. Palmer et al., IAU Circ., 6577 (1997).

[3] E. Costa et al., Nature (London) 387, 783 (1997).

[4] A. Guarnieri et al., Astron. Astrophys. 328, L13 (1997).

[5] M. J. Rees and P. Mészáros, Mon. Not. R. Astron. Soc. 258, 41p (1992).

[6] J. I. Katz, Astrophys. J. 422, 248 (1994).

[7] R. Sari, R. Narayan, and T. Piran, Astrophys. J. 473, 204 (1996).

[8] G. J. Fishman et al., Astrophys. J. Suppl. 92, 229 (1994).

[9] K. Hurley et al., Nature (London) 372, 652 (1994).

[10] C. Meegan et al., IAU Circ., 6518 (1996).

[11] V. Connaughton et al., in Proceedings of the 18th Texas Symposium on Relativistic Astrophysics (N. Y. Acad. Sci., New York, to be published).

[12] M. Koshut et al., Astrophys. J. 452, 145 (1995).

[13] R. Sari and T. Piran, Astrophys. J. 485, 270 (1997).

[14] J. I. Katz, Astrophys. J. 490, 633 (1997).

[15] J. I. Katz, Astrophys. J. Lett. 432, L107 (1994).

[16] M. Tavani, Astrophys. Space Sci. 231, 181 (1995).

[17] E. Cohen, J. I. Katz, T. Piran, R. Sari, R. D. Preece, and D. L. Band, Astrophys. J. 488, 330 (1997).

[18] H. Pedersen et al., Astrophys. J. (to be published) [Report No. astro-ph/9710322].

[19] L. Piro et al., Astron. Astrophys. (to be published) [Report No. astro-ph/9710355]. 